\title{
The critical renormalized coupling constants in the symmetric phase of the Ising models
}

\author{
Jae -Kwon Kim \\ School of Physics, Korea Institute for Advanced Study, \\ 207-43 Cheongryangri-dong, Dongdaemun-gu, Seoul 130-012, Korea
}

Using a novel finite size scaling Monte Carlo method, we calculate the four, six and eight point renormalized coupling constants defined at zero momentum in the symmetric phase of the three dimensional Ising system. The results of the $2 \mathrm{D}$ Ising system that were directly measured are also reported. Our values of the six and eight point coupling constants are significantly different from those obtained from other methods.

\section{INTRODUCTION}

The Hamiltonian of the Ising ferromagnet is given by

$$
H=-\sum_{<i, j>} S_{i} S_{j}
$$

where the Ising spin at site $i, S_{i}$, can take either 1 or -1 and the sum is over all the nearest neighbors of the lattice. It is well known that the critical behavior of the $\mathrm{D}$ dimensional Ising model can be described by the $\mathrm{D}$ dimensional Euclidean scalar field theory the Hamiltonian of which is given by

$$
H=\int d^{D} x\left[\frac{1}{2}(\nabla \phi(x))^{2}+\frac{1}{2} m_{0}^{2} \phi(x)^{2}+\frac{g_{0}}{4 !} \phi(x)^{4}\right],
$$

where $m_{0}$ and $g_{0}$ are respectively the bare mass and coupling constant defined in the absence of critical fluctuations of the fields. Near the criticality $m_{0}$ is a linear measure of temperature, and we denote by $m_{0 c}$ the value for which the theory becomes critical. As the fluctuations become strong renormalizations of the mass, coupling constant and fields are necessary, and the long distance behavior is no longer described by the bare potential but by the effective potential which is generally of more complicated functional form than the bare one. In statistical physics the effective potential represents the free energy density as a function of order parameter (expectation value of the renormalized field), and is used to determine the equation of state.

After small-renormalized-field expansion of the effective potential, its coefficients are directly related to the renormalized coupling constants (RCCs) defined at zeromomentum. In terms of the expectation value of the renormalized field, $\varphi_{R}$, the effective potential in the $3 \mathrm{D}$ symmetric phase may be written as

$$
V_{e f f}\left(\varphi_{R}\right)=\frac{1}{2} m_{R}^{2} \varphi_{R}^{2}+\frac{1}{4 !} m_{R} g_{R}^{(4)} \varphi_{R}^{4}+
$$

$$
\frac{1}{6 !} g_{R}^{(6)} \varphi_{R}^{6}+\frac{1}{8 !} \frac{g_{R}^{(8)}}{m_{R}} \varphi_{R}^{8}+\ldots
$$

where $m_{R}$ and $g_{R}^{(N)}$ represent respectively the renormalized mass (inverse correlation length $\xi$ ) and the (dimensionless) $N$-point RCC defined at zero momentum.

The formal expression of $g_{R}^{(N)}$ can be obtained by calculating the $N$-th derivative of the effective potential with respect to the average value of the renormalized field. Necessary elements for the calculations are the well-known relations

$$
\frac{d V_{e f f}}{d \varphi}=J, \quad \varphi=\frac{1}{V} \frac{d W[J]}{d J}, \text { and } \varphi=Z_{\varphi}^{1 / 2} \varphi_{R} .
$$

Here $\varphi, V, W$, and $Z_{\varphi}$ are respectively the expectation value of the bare field $\phi$, the volume of the system, the generating functional for connected Green functions in the presence of the external field $J$, and the field strength renormalization factor given by $Z_{\varphi}=\chi m_{R}^{2}$ with $\chi$ denoting magnetic susceptibility. The expressions in $3 \mathrm{D}$ are

$$
\begin{aligned}
& g_{R}^{(4)}=-\left(Z_{\varphi}^{2} / m_{R}\right) W_{2}^{-4} W_{4} \\
& g_{R}^{(6)}=-Z_{\varphi}^{3} W_{2}^{-6}\left[W_{6}-10 W_{4}^{2} W_{2}^{-1}\right] \\
& g_{R}^{(8)}=-m_{R} Z_{\varphi}^{4} W_{2}^{-8} \\
& \quad \times\left[W_{8}-56 W_{6} W_{4} W_{2}^{-1}+280 W_{4}^{3} W_{2}^{-2}\right],
\end{aligned}
$$

where $W_{N}$ is the Fourier transformed $N$-point connected Green function at zero momentum. In $2 \mathrm{D} \varphi_{R}$ is dimensionless, so that the expressions of the $g_{R}^{(2 N)}$ are given by the above equations with additional division of the $m_{R}^{2}$ term.

Each RCC has a universal zon-zero finite value as the theory becomes critical, provided the hyperscaling relation holds. To be more specific, we take thermodynamic limit first and then take the limit of the thermodynamic correlation length becoming divergent (that is, the limit $\left.m_{0} \rightarrow m_{0 c}\right)$. The corresponding value in this limit may be called as critical RCC. The determination of the accurate value of the critical four-point $\operatorname{RCC}\left(\tilde{g}_{R}^{(4)}\right)$ is particularly important since all other universal quantities can be given in terms of it.

In this work we are mainly concerned with the Monte Carlo calculation of the critical RCCs in the symmetric phase of the three dimensional (3D) Ising model. (From here on the notation of RCC will be used without the subscript $R$ when it is used in context of size dependence.) 
This has been a subject of many studies ipcluding a variety of quantum field the retic approachs 4 , high temperature series expansions 5 , exact renormalization oroup flow techniques 10, and Monte Carlo simulations11, 22,20 . It is known that standard direct Monte Carlo measurements of the thermodynamic critical RCCs suffer from enormous statistical noise 11 . Other Monte Carlo study is based on the finding of the probability distribution of the order parameter in the external field at various temperatures 12. We employ a novel finite size scaling (FSS) technique 13 .14 combined with single cluster flipping Monte Carlo algorithm17. Our preliminary results were reported in Ref.( 15). Here we report the result of our updated Monte Carlo measurements that has been increased more than one magnitude of order. We also report our results measured at criticality that is useful to determine the correction to scaling in the RCCs as well as results in the $2 \mathrm{D}$ system.

\section{THE MONTE CARLO METHOD}

For the calculations of the RCC one needs to calculate $\xi, \chi$, and $W_{2 N}$. With periodic boundary condition imposed on the lattice, $\chi$ and $W_{2 N}$ can be expressed in terms of the expectation values of various powers of the sum of the spin over all the lattice site $S \equiv \sum_{i} S_{i}$. For example,

$$
\chi=<S^{2}>/ L^{D}, \quad W_{4}=\left(<S^{4}>-3<S^{2}>^{2}\right) / L^{D},
$$

where $L$ is the linear size of the lattice. $\xi$ can be very accurately determined using the stapdard second moment formula 16. The cluster algorithms 17 of the Monte Carlo simulation have been extremely efficient for many problems of critical phenomena. It is now an easy task to obtain Monte Carlo data of typical physical quantities such as $\chi$ and $\xi$ with relative statistical errors less than 0.1 percent at a temperature arbitrarily close to criticality. Nevertheless, it turns out that accurate Monte Carlo measurement of higher order RCC is problematic in some cases. The problem stems basically from the fact that the higher order RCC is given as a multiplication of a huge number with a tiny one. The former is given typically by some power of $\frac{L}{\xi_{L}}$, for instance $\left(\frac{L}{\xi_{L}}\right)^{9}$ for the $g_{R}^{(8)}$ of the $3 \mathrm{D}$ system, and the latter comes from the combination of the $W_{2 N}$ that turns out to be extremely sensitive to statistical noises. The noises increase rapidly with increasing temperature and increasing value of $L / \xi_{L}$.

To illustrate we measured various physical quantities at an arbitrary (inverse) temperature in the scaling regime of the $2 \mathrm{D}$ Ising model with increasing linear size of the lattice L. Table(II) clearly shows that the statistical error in the measured values of $g_{R}^{(2 N)}$ increases with $N$ for any given value of $\mathrm{L}$. For $\mathrm{L}=80$, for example, the relative statistical errors of $\xi_{L}, \chi_{L}, g_{L}^{(4)}, g_{L}^{(6)}, g_{L}^{(8)}$ are respectively $0.08,0.1,0.7,3.1,5.3$ percents. We observe clearly that all the variables except for $g_{L}^{(8)}$ are monotonically increasing functions of $L$, up to $\mathrm{L}=80$ where $L / \xi_{L} \simeq 6.7$. For further increasing $L$ the values do not vary within the statistical errors. We recall that the wellknown exact thermodynamic value of the $\xi$ at this $\beta$ is $11.9055 \ldots$, which manifests itself at $\mathrm{L}=80$ already within the statistical errors. The size independent value thus corresponds to the thermodynamic value. It is also very clear that the size dependence becomes rapidly weaker with increasing $L$ for all the variables we consider here. This is expected to be the case for any physical variable having well-defined thermodynamic value. For the $g_{L}^{(8)}$, however, due to the large statistical noises is it hard to draw a definite conclusion on the size dependence. Nevertheless, it is very natural to expect that $g_{L}^{(8)}$ would follow similar size dependence as $g_{L}^{(4)}$ and $g_{L}^{(6)}$.

To compute the critical RCC it is required to obtain thermodynamic values as $\beta \rightarrow \beta_{c}$. For the case of the $2 \mathrm{D}$ Ising model, we will not follow that procedure. Instead, we will report the results of measurements at criticality and try to find out possible effect of the correction to scaling. The illustration in the case of the 2D Ising model will be useful in connection with the study of the $3 \mathrm{D}$ case. Note that the relation in the scaling regime

$$
g_{R}^{(2 N)}(t) \sim t^{D \nu-2 \Delta_{2 N}+\gamma},
$$

translates into

$$
g_{L}^{(2 N)}(t=0) \sim L^{\left(D \nu-2 \Delta_{2 N}+\gamma\right) / \nu},
$$

at criticality. Hence the hyperscaling relation $D \nu-$ $2 \Delta_{2 N}+\gamma=0$ implies the invariance of $g_{L}^{(2 N)}(t=0)$ with respect to $L$. Conversely, from the (weak) dependence of of $g_{L}^{(2 N)}(t=0)$ can one infer the correction to scaling in the scaling regime. Computing RCC by taking the limit $t \rightarrow 0$ first and then take the limit $L \rightarrow \infty$ is equivalent to the computation of $g_{L}^{(2 N)}(t=0)$. It should be stressed that $g_{L}^{(2 N)}(t=0)$ basically represents the apposite of thermodynamic limit for a non-zero $t \rightarrow 0$ (that is $L \rightarrow 0$ limit for $\xi$ becomes huge but not infinite) and has nothing to do with the lower-bound of critical RCd21.

We also report so called 6-th and 8-th order cumulant ratios at criticality that are denoted by $U_{L}^{(6)}$ and $U_{L}^{(8)}$ respectively. These are part of $W_{6}$ and $W_{8}$, and have already been available for the $2 \mathrm{D}$ Ising medel based on umbrella sampling method of the Monte Carlo simulation22. Since our code is generic in dimension, the comparison with the result from different Monte Carlo method for these higher order cumulant ratios having severe statistical noises may be useful to check against any unexpected possible systematic errors in our Monte Carlo measurements. For example, $U_{L}^{(8)}$ is expressed by

$$
\begin{aligned}
U_{L}^{(8)}= & \left(<S^{8}>-28<S^{6}><S^{2}>-35<S^{4}>^{2}+\right. \\
& \left.420<S^{4}><S^{2}>^{2}-630<S^{2}>^{4}\right) /<S^{2}>^{4}
\end{aligned}
$$


The results are summerized in Table(II). Our results of $U_{L}^{(2 N)}$ for $\mathrm{N}=2,3$, and 4 are respectively $1.8318(5)$, 13.93(1), and -226.0(1), which may be compared with 1.834, 13.96, and -226.6 reported in Ref. 22. Currently accepted value of $U_{L}^{(4)}$ is $1.832(1)$. We observe that $g_{L}^{(2 N)}(t=0)$ has no $L$ dependence at least for $L \geq 40$, confirming the hyperscaling and no significant correction to scaling. We therefore lead to the conclusion that the thermodynamic values of RCC measured at $\beta=0.420$ may well be regarded as the critical values.

Regarding size dependence we observe similar feature in the 3D Ising model as in the 2D case. Our data are summerized in Table(III). For the $\beta=0.217$ and 0.220 we observe no size dependence (within the statistical errors) for the $\xi$ and $\chi$ beyond $\mathrm{L}=32$ and 60 respectively. This roughly corresponds to $L / \xi_{L} \simeq 5.5$. We expect that this is the case even for the RCCs, although it was almost impossible to get precise measurements of the thermodynamic values of $g_{L}^{(6)}$ and $g_{L}^{(8)}$ for this value of $L / \xi_{L}$. For example, for $\beta=0.217$ and $L=32$ we generated about $10^{9}$ single cluster sweeps, but error bars are larger than the mean value for the $g_{L}^{(8)}$. The values of $g_{L}^{(6)}$ for $L \geq 32$ also seem to be unreliable in view of the generic feature of the weaker size dependence with larger $L$.

It thus appears that Monte Carlo computation of the critical RCCs relying on direct brute force measurements is prohibitively difficult. In order to overcome the difficulty closer $T_{c}$, we make use of a new FSS function $\mathcal{Q}_{A}(x(L, t)) 13,14$, defined by the expression

$$
A_{L}(t)=A(t) \mathcal{Q}_{A}(x(L, t)), \quad x(L, t) \equiv \xi_{L}(t) / L .
$$

Here $A_{L}(t)$ represents the quantity $A$ measured on a finite lattice of linear size $L$ at a reduced temperature $t$, with its corresponding thermodynamic value $A(t)$. What Eq. 12) states is that the size dependence of a physical quantity $A$ is given as a function of the scaling variable $x$. As a result, the ratio of $L$ to $\xi_{L}$ beyond which thermodynamic limit is reached is independent of the temperature, that turned out to be approximately 5.5 for the 3D Ising model.

The FSS technique is especially useful for our purpose, because it enables us to extract accurate thermodynamic values based on the Monte Carlo measurements with much smaller lattices. We just outline the single step FSS extrapolation technique used in this work. For a detailed explanation, we refer the readers to Ref. 13, 14 .

1. For a certain $t_{0}$, measure $A_{L}\left(t_{0}\right)$ and $x\left(L, t_{0}\right)=$ $\xi_{L}\left(t_{0}\right) / L$ for increasing $\mathrm{L}$.

2. Determine the thermodynamic value at the temperature $A\left(t_{0}\right)$ by measuring $A_{L}\left(t_{0}\right)$ which is $L$ independent.

3. Fit $\left(x\left(L, t_{0}\right), A_{L}\left(t_{0}\right) / A\left(t_{0}\right)\right)$ data to a suitable functional form. In this work we used the ansatz,

$$
\mathcal{Q}(x)=1+c_{1} x+c_{2} x^{2}+c_{3} x^{3}+c_{4} x^{4}
$$

4. For any other $t$, choose a suitable L, measure the value of $x(L, t) \equiv \xi_{L} / L$ and $A_{L}(t)$, and interpolate $\mathcal{Q}(x(L, t))$.

5. Extract $A(t)$ by plugging $A_{L}(t)$ and $\mathcal{Q}(x(L, t))$ into Eq.(12).

The smallest value of $\mathrm{L}$ we considered for the FSS method is 20 .

\section{RESULT AND DISCUSSION}

Our choice of $\beta_{0}$ is $\beta_{0}=0.220$. We infer from the generic feature of the size dependence observed for the 3D Ising model that thermodynamic limit of the RCCs are reached for $L / \xi_{L} \gtrsim 5.5$. We thus get $g_{R}^{(4)}=24.5(4)$ and $g_{R}^{(6)}=1983(148)$ at this $\beta$. Fitting the data to the ansatz Eq.(13), we get

$c_{1}=2.338, \quad c_{2}=-15.768, \quad c_{3}=19.770, \quad c_{4}=-5.123$

$c_{1}=3.225, \quad c_{2}=-25.046, \quad c_{3}=42.485, \quad c_{4}=-23.177$

for the scaling function $\mathcal{Q}(x)$ of $g^{(4)}$ and $g^{(6)}$ respectively. Using the scaling function we calculated the thermodynamic values of the 4-point and 6-point RCCs for all the $\mathrm{L}$ from 36 to 80 in the Table( $(\mathrm{IV})$. The result at each $\mathrm{L}$ is in reasonably good agreement. For example, we get $g_{R}^{(4)}=24.3(1), 24.4(4), 24.1(1), 23.9(1), 23.9(1), 24.1(3)$, and $23.9(2)$ for each $L$ from the $\mathrm{L}=36$ through the $\mathrm{L}=80$ in the table. For $g_{R}^{(6)}$ we get 1919(11), 1939(20), 1939(21), $1915(10), 1906(17), 1917(70)$, and 1897(49). The invariance of the thermodynamic $\mathrm{RCC}$ with respect to the choice of $L$ is a numerical proof of the FSS for the variables (see Figure(11) and (2)). We usually extracted the thermodynamic value for several different choices of $L$ for a given temperature, and took the average. Our net results from $\beta=0.217$ to $\beta=0.2213$ are found in Table(VI). It is observed that both $g_{R}^{(4)}$ and $g_{R}^{(6)}$ tend to decrease mildly as $\beta \rightarrow \beta_{c}$. In this work we assume the widely accepted correction to scaling exponent $\theta \simeq 0.5$ and $\beta_{c}=0.22165419$. By fitting our data in Table( $(\mathrm{VI})$ to

$$
g_{R}^{(2 N)}(t)=\tilde{g}_{R}^{(2 N)}\left(1+a_{2 N} t^{0.5}\right),
$$

we obtain the critical $\mathrm{RCC}$ which reads

$$
\begin{aligned}
& \tilde{g}_{R}^{(4)}=23.6(2) \\
& \tilde{g}_{R}^{(6)}=1879(50)
\end{aligned}
$$

Our results of $g_{L}^{(2 N)}(t=0)$ are found in Table( $(\mathbb{\nabla})$. It is observed that both of $U_{L}^{(4)}$ and $\xi_{L} / L$ have tendency of very mild decreasing with increasing $L$. All the $g_{L}^{(2 N)}(t=0)$ show remarkable invariance with respect to increasing $\mathrm{L}$ at least for $L \geq 30$. In other words, they do not show the effect of correction to scaling observed 
in the scaling regime. There may be a few possible interpretations for the discrepancy. First let us remind that Eq.(14) translates into

$$
g_{L}^{(2 N)}(t=0)=\tilde{g}^{(2 N)}(t=0)\left(1+b_{2 N} L^{-\omega}\right), \quad(\omega>0),
$$

and that the coefficient $b_{2 N}$ may happen to be very small. The second possibility is that currently accepted $\beta_{c}$ might be slightly underestimated: At exact criticality it is expected that $\xi_{L} / L$ remains an exact constant irrespective of the presence of the correction, which seems not to be the case at $\beta=0.221654$.

Our current result at criticality may be summerized as follows.

$$
\begin{aligned}
& \tilde{g}^{(4)}(t=0)=5.36(2) \\
& \tilde{g}^{(6)}(t=0)=154.6(1.1) \\
& \tilde{g}^{(8)}(t=0)=1.035(10) \times 10^{4} .
\end{aligned}
$$

It is impossible to apply the FSS method for the $g_{R}^{(8)}$ due to its large error bars. Nevertheless, it is highly likely that at least $\tilde{g}_{R}^{(8)}$ is of order $10^{5}$. (see Table([II).) Also we have reliable data at $\beta=0.2213$ showing $g_{L}^{(8)} \simeq$ $1.13(3) \times 10^{5}$ already for $L / \xi_{L} \simeq 2.7$. Furthermore it is observed that the ratio of critical RCCs to $g_{L}^{(2 N)}(t=0)$ tends to increase with $N$; The ratio is roughly 4.4 and 12.2 for $\mathrm{N}=2$ and 3 respectively for the $3 \mathrm{D}$. We also note that $L$ dependence of $g_{L}^{(8)}(t=0)$ is quanitatively the same as that of the other RCCs, indicating that the correction to scaling for $g_{R}^{(8)}(t)$ is as mild as the other RCCs. All the evidence almost undoubtedly point out that the critical $g_{R}^{(8)}$ is much larger than those estimated by other methods. Our crude estimate of $\tilde{g}_{R}^{(8)}$ reads

$$
\tilde{g}_{R}^{(8)} \simeq 1.4(3) \times 10^{5} .
$$

Our value of $\tilde{g}_{R}^{(4)}$ is in reasonable agreement with other estimates. The agreement is especially good with the result from high-temperature expansion 5 and the field theoretic treatment 3 . However, the agreement becomes worse as $N$ increases: The results of $\tilde{g}_{R}^{(6)}$ from previous studies are within the range $860 \lesssim \tilde{g}_{R}^{(6)} \lesssim 1515$. The closest result to ours, $\tilde{g}_{R}^{(6)} \simeq 1515$, obtained from the previous Monte Carlo method 2 is remarkable because it is obtained not by the measurement of RCC but by the Monte Carlo measurement of the probability distribution of order parameter assuming the thermodynamic limit for $L / \xi_{L} \simeq 4$. We conjecture that this result would agree much better with our estimate if the same thermodynamic limit as in this work would have been taken. The values of $\tilde{g}_{R}^{(8)}$ from previous studies ranges from $2.9 \times 10^{4}$ to $3.5 \times 10^{4}$, which is at least four times smaller than our estimate. Although it was pointed out that longer series terms are necessary for more accurate estimate of higher order critical RCC from the high temperature series expansion method, it remains mysterious why previous studies based on different methods give rise to reasonably consistent results among them. Nevertheless, in view of limitations of almost all the methods used to study this subject, it is fair to say that the issue is still open to further studies.

Our results of the critical RCCs for 2D Ising model obtained assuming negligibly small correction to scaling read

$$
\begin{aligned}
& \tilde{g}_{R}^{(4)}=14.7(2) \\
& \tilde{g}_{R}^{(6)}=850(25) \\
& \tilde{g}_{R}^{(8)}=8.9(5) \times 10^{4} .
\end{aligned}
$$

The only result on the high order RCCs we are aware of is the $\varepsilon$ expansion study 2 ;

$$
\tilde{g}_{R}^{(6)}=794, \quad \tilde{g}_{R}^{(8)} \simeq 8.2(2) \times 10^{4},
$$

which agree reasonably well with ours.

The results at criticality are summerized as

$$
\begin{aligned}
& \tilde{g}^{(4)}(t=0)=2.239(7) \\
& \tilde{g}^{(6)}(t=0)=29.34(20) \\
& \tilde{g}^{(8)}(t=0)=947(10)
\end{aligned}
$$

We wish to thank Maxim Tsypin for many communications.

TABLE I. Size dependence of various physical quantities at $\beta=0.420$ up to $\mathrm{L}=100$ for the $2 \mathrm{D}$ Ising system.

\begin{tabular}{llllll}
\hline \hline $\mathrm{L}$ & $\xi_{L}$ & $\chi_{L}$ & $g_{L}^{(4)}$ & $g_{L}^{(6)}$ & $g_{L}^{(8)}$ \\
\hline 20 & $9.664(8)$ & $116.1(1)$ & $6.29(1)$ & $218.46(1.32)$ & $17871(140)$ \\
30 & $10.977(9)$ & $162.5(1)$ & $8.95(1)$ & $414.8(2.3)$ & $43532(313)$ \\
40 & $11.53(1)$ & $186.5(2)$ & $11.26(1)$ & $615.1(4.0)$ & $71443(615)$ \\
50 & $11.78(1)$ & $197.4(2)$ & $12.83(3)$ & $740.2(5.5)$ & $85462(879)$ \\
60 & $11.84(1)$ & $201.3(2)$ & $13.79(4)$ & $794.8(6.2)$ & $87919(1185)$ \\
70 & $11.88(1)$ & $203.1(2)$ & $14.20(6)$ & $825.1(10.0)$ & $89824(2315)$ \\
80 & $11.91(1)$ & $203.9(2)$ & $14.77(10)$ & $850.4(26.1)$ & $89521(4765)$ \\
90 & $11.91(2)$ & $204.3(2)$ & $14.85(12)$ & $858.8(24.2)$ & $90722(7451)$ \\
100 & $11.90(2)$ & $204.4(3)$ & $14.60(16)$ & $846(38)$ & $88165(9575)$ \\
\hline \hline
\end{tabular}

TABLE II. Size dependence of various physical quantities at criticality $\beta_{c}=\ln (\sqrt{2}+1) / 2$ up to $\mathrm{L}=100$ for the $2 \mathrm{D}$ Ising system.

\begin{tabular}{lllllll}
\hline \hline $\mathrm{L}$ & $U_{L}^{(4)}$ & $U_{L}^{(6)}$ & $U_{L}^{(8)}$ & $g_{L}^{(4)}$ & $g_{L}^{(6)}$ & $g_{L}^{(8)}$ \\
\hline 20 & $1.8324(6)$ & $13.94(1)$ & $-226.1(2)$ & $2.227(7)$ & $29.03(18)$ & $933(9)$ \\
40 & $1.8321(6)$ & $13.94(1)$ & $-226.1(2)$ & $2.237(8)$ & $29.28(21)$ & $945(10)$ \\
60 & $1.8317(5)$ & $13.93(1)$ & $-225.9(1)$ & $2.241(7)$ & $29.37(18)$ & $949(9)$ \\
80 & $1.8318(5)$ & $13.93(1)$ & $-226.0(1)$ & $2.240(6)$ & $29.36(16)$ & $948(8)$ \\
100 & $1.8316(6)$ & $13.93(1)$ & $-226.0(1)$ & $2.239(7)$ & $29.33(18)$ & $947(9)$ \\
\hline \hline
\end{tabular}


TABLE III. Size dependence of the various physical quantities at $\beta=0.217$ (the upper part) and $\beta=0.220$ (the lower part). Note that the $g_{L}^{(8)}$ data for $L / \xi_{L} \geq 5.7$ become unreliable due to huge error bars and in view of weaker size dependence for larger $L$. This appears to be case even to the $g_{L}^{(6)}$ for $L=36$.

\begin{tabular}{lllllc}
\hline \hline $\mathrm{L}$ & $\xi_{L}$ & $\chi_{L}$ & $g_{L}^{(4)}$ & $g_{L}^{(6)}$ & $g_{L}^{(8)} \times 10^{-4}$ \\
\hline 8 & $3.93(0)$ & $59.49(6)$ & $9.86(2)$ & $492.5(1.9)$ & $5.45(3)$ \\
12 & $4.85(1)$ & $94.16(12)$ & $13.59(3)$ & $852(4)$ & $10.8(1)$ \\
16 & $5.299(1)$ & $114.54(5)$ & $17.71(2)$ & $1277(3)$ & $15.77(9)$ \\
20 & $5.488(3)$ & $124.20(7)$ & $21.07(5)$ & $1562(9)$ & $15.5(3)$ \\
24 & $5.573(2)$ & $128.40(5)$ & $23.16(8)$ & $1735(22)$ & $15.5(9)$ \\
28 & $5.605(4)$ & $130.06(11)$ & $24.72(11)$ & $1887(53)$ & $14.6(3.8)$ \\
32 & $5.622(2)$ & $130.87(5)$ & $24.88(14)$ & $2190(86)$ & $13.3(16.9)$ \\
36 & $5.619(19)$ & $130.96(14)$ & $25.64(16)$ & $2860(191)$ & $83.3(29.1)$ \\
\hline 16 & $7.85(2)$ & $228.8(7)$ & $9.52(5)$ & $452.1(4.6)$ & - \\
20 & $8.85(2)$ & $298.1(7)$ & $11.43(5)$ & $625.6(5.1)$ & - \\
24 & $9.56(2)$ & $351.8(1.1)$ & $13.44(7)$ & $814.7(8.2)$ & - \\
30 & $10.20(3)$ & $407.1(1.1)$ & $16.5(1)$ & $1123(16)$ & - \\
36 & $10.56(2)$ & $439.2(1.2)$ & $19.2(1)$ & $1372(27)$ & - \\
40 & $10.68(3)$ & $455.2(1.5)$ & $21.4(2)$ & $1501(56)$ & - \\
50 & $10.83(3)$ & $467.9(1.3)$ & $23.7(3)$ & $1795(109)$ & - \\
60 & $10.89(3)$ & $472.3(1.8)$ & $24.5(4)$ & $1983(148)$ & - \\
70 & $10.90(3)$ & $473.0(1.1)$ & $25.8(1.6)$ & - & - \\
\hline \hline
\end{tabular}

TABLE IV. Size dependence of the various physical quantities at $\beta=0.221$ up to $L / \xi_{L} \simeq 4.11$.

\begin{tabular}{lllllc}
\hline \hline $\mathrm{L}$ & $\xi_{L}$ & $\chi_{L}$ & $g_{L}^{(4)}$ & $g_{L}^{(6)}$ & $g_{L}^{(8)} \times 10^{-4}$ \\
\hline 20 & $10.95(2)$ & $426.7(1.4)$ & $7.6(1)$ & $301(3)$ & $2.67(4)$ \\
28 & $13.93(6)$ & $703.4(4.0)$ & $9.2(1)$ & $423(8)$ & $4.3(1)$ \\
36 & $15.98(6)$ & $944.2(4.7)$ & $11.2(1)$ & $600(10)$ & $6.6(2)$ \\
40 & $16.74(5)$ & $1045.5(4.3)$ & $12.4(1)$ & $710(13)$ & $8.2(2)$ \\
48 & $17.91(3)$ & $1210(3)$ & $14.59(6)$ & $920(8)$ & $10.8(2)$ \\
56 & $18.60(1)$ & $1316(1)$ & $16.74(5)$ & $1116(7)$ & $12.2(2)$ \\
64 & $19.03(2)$ & $1386(2)$ & $18.66(7)$ & $1297(13)$ & $13.4(4)$ \\
72 & $19.28(5)$ & $1426(6)$ & $20.47(29)$ & $1465(57)$ & $13.3(2.6)$ \\
80 & $19.46(2)$ & $1458(2)$ & $21.36(16)$ & $1580(82)$ & - \\
\hline \hline
\end{tabular}

TABLE V. Size dependence of various physical quantities at criticality $\beta_{c}=0.221654$ up to $\mathrm{L}=80$ for the $3 \mathrm{D}$ Ising system. Here $U_{L}^{(4)}$ represents fourth-order cumulant ratio.

\begin{tabular}{lllllll}
\hline \hline $\mathrm{L}$ & $U_{L}^{(4)}$ & $\xi_{L}$ & $\chi_{L}$ & $g_{L}^{(4)}$ & $g_{L}^{(6)}$ & $g_{L}^{(8)} \times 10^{-4}$ \\
\hline 20 & $1.418(1)$ & $12.80(1)$ & $545.1(5)$ & $5.40(1)$ & $157.4(8)$ & $1.07(1)$ \\
30 & $1.409(1)$ & $19.20(2)$ & $1215(1)$ & $5.37(1)$ & $155.3(7)$ & $1.04(1)$ \\
40 & $1.408(2)$ & $25.65(4)$ & $2148(4)$ & $5.34(2)$ & $153.5(1.1)$ & $1.03(1)$ \\
50 & $1.403(1)$ & $31.98(4)$ & $3313(4)$ & $5.36(2)$ & $154.4(1.0)$ & $1.03(1)$ \\
60 & $1.401(2)$ & $38.33(6)$ & $4742(10)$ & $5.37(2)$ & $155.1(1.1)$ & $1.04(1)$ \\
70 & $1.399(3)$ & $44.69(8)$ & $6418(19)$ & $5.38(2)$ & $155.2(1.2)$ & $1.04(1)$ \\
80 & $1.398(2)$ & $51.13(9)$ & $8351(19)$ & $5.36(2)$ & $154.1(1.3)$ & $1.03(1)$ \\
\hline \hline
\end{tabular}


TABLE VI. Thermodynamic values of the four and six point RCC extracted by the FSS technique for some temperatures over $0.217 \leq \beta \leq 0.2213$. Here the quoted errors are obtained ignoring the statistical errors in the estimate of the thermodynamic values at $\beta=0.220$. Crudely speaking, increases in the estimate of beta $=0.220$ would lead to more or less similar amount of increase in the estimate for other temperatures.

\begin{tabular}{|c|c|c|c|c|c|c|}
\hline$\beta$ & 0.217 & 0.219 & 0.220 & 0.2206 & 0.2210 & 0.2213 \\
\hline$\overline{g_{R}^{(4)}}$ & $25.2(2)$ & $24.7(2)$ & $24.5(0)$ & $24.2(2)$ & $24.2(2)$ & $24.1(2)$ \\
\hline$g_{R}^{(6)}$ & $2034(69)$ & $2063(46)$ & $1983(0)$ & $1988(81)$ & $1919(51)$ & $1943(42)$ \\
\hline
\end{tabular}


FIG. 1. $\mathcal{Q}_{g^{4}}$ calculated for $\beta=0.220$ and 0.221 . The two data set collapse unto a single universal curve, showing a numerical evidence for the FSS Eq. 12 for $g_{R}^{(4)}$.

FIG. 2. The same as in Figure(1) but for $\mathcal{Q}_{g^{6}}$.

${ }^{1}$ C. M. Bender et al, Phys. Rev. Lett. 45, 501 (1980); C. M. Bender and S. Boettcher, Phys. Rev. D 48, 4919 (1993)

${ }^{2}$ A. I. Sokolov, Fiz, Tverd. Tela (Solid State Phys.) 38, 640 (1997); A. I. Sokolov, V. A. Ulḱov, and E. V. Orlov, Phys. Lett. A 227, 255 (1997)

${ }^{3}$ R. Guida and J. Zinn-Justin, Nucl. Phys. B 489, 626 (1997)

${ }^{4}$ M. Campostrini, A. Pelissetto, P. Rossi and E. Vicari, Nucl. Phys. B 459, 207 (1996)

${ }^{5}$ P. Butera and M. Comi, Phys. Rev. B 54, 15828 (1996); ibid, Phys. Rev. B 58, 11552 (1998)

${ }^{6}$ T. Reisz, Nucl. Phys. B 450, 569 (1995)

${ }^{7}$ S. Y. Zinn, S. N. Lai, and M. E. Fisher, Phys. Rev. E 54, $1176(1996)$

${ }^{8}$ C. Bagnuls and C. Bervillier, Phys. Rev. B 41, 402 (1990)

${ }^{9}$ J. Berges, N. Tetradis, and C. Wetterich, Phys. Rev. Lett. 77, 873 (1996)

10 T. Morris, Nucl. Phys. B 495, 4777 (1997)

${ }^{11}$ J. F. Wheater, Phys. Lett. B 136, 402 (1984)

${ }^{12}$ M. M. Tsypin, Phys. Rev. Lett. 73, 2015 (1994)

${ }^{13}$ J.-K. Kim, Phys. Rev. D 50, 4663 (1994); for a slightly different form of FSS, see S. Caracciolo, R. G. Edwards, S. J. Ferreira, A. Pelissetto, and A. D. Sokal, Phys. Rev. Lett. 74, 2969 (1995)

${ }^{14}$ J.-K. Kim, A. J. F. de Souza, and D. P. Landau, Phys. Rev. E 54, 2291 (1996)

${ }^{15}$ J.-K. Kim and D. P. Landau, Nucl. Phys. B (Proc. Suppl.) 53, 706 (1997)

${ }^{16}$ See for example, Ref. $(14$.

${ }^{17}$ U. Wolff, Phys. Rev. Lett. 62, 361 (1989); R. H. Swendsen and J.-S. Wang, Phys. Rev. Lett. 58, 86 (1987)

${ }^{18}$ A. Pelissetto and E. Vicari, Nucl. Phys. B 522 [FS], 605 (1998)

19 See for example, R. Guida and J. Zinn-Justin, J. Phys. A: Math. Gen. 31, 8103 (1998) and references therein.

20 J.-K. Kim and A. Patrascioiu, Phys. Rev. D 47, 2588 (1993)

${ }^{21}$ G. A. Baker, Jr and N. Kawashina, Phys. Rev. Lett. 75, 994 (1995); J.-K. Kim, Phys. Rev. Lett. 76, 2402 (1996)

${ }^{22}$ K. K. Mon, Phys. Rev. B 55, 38 (1997) 


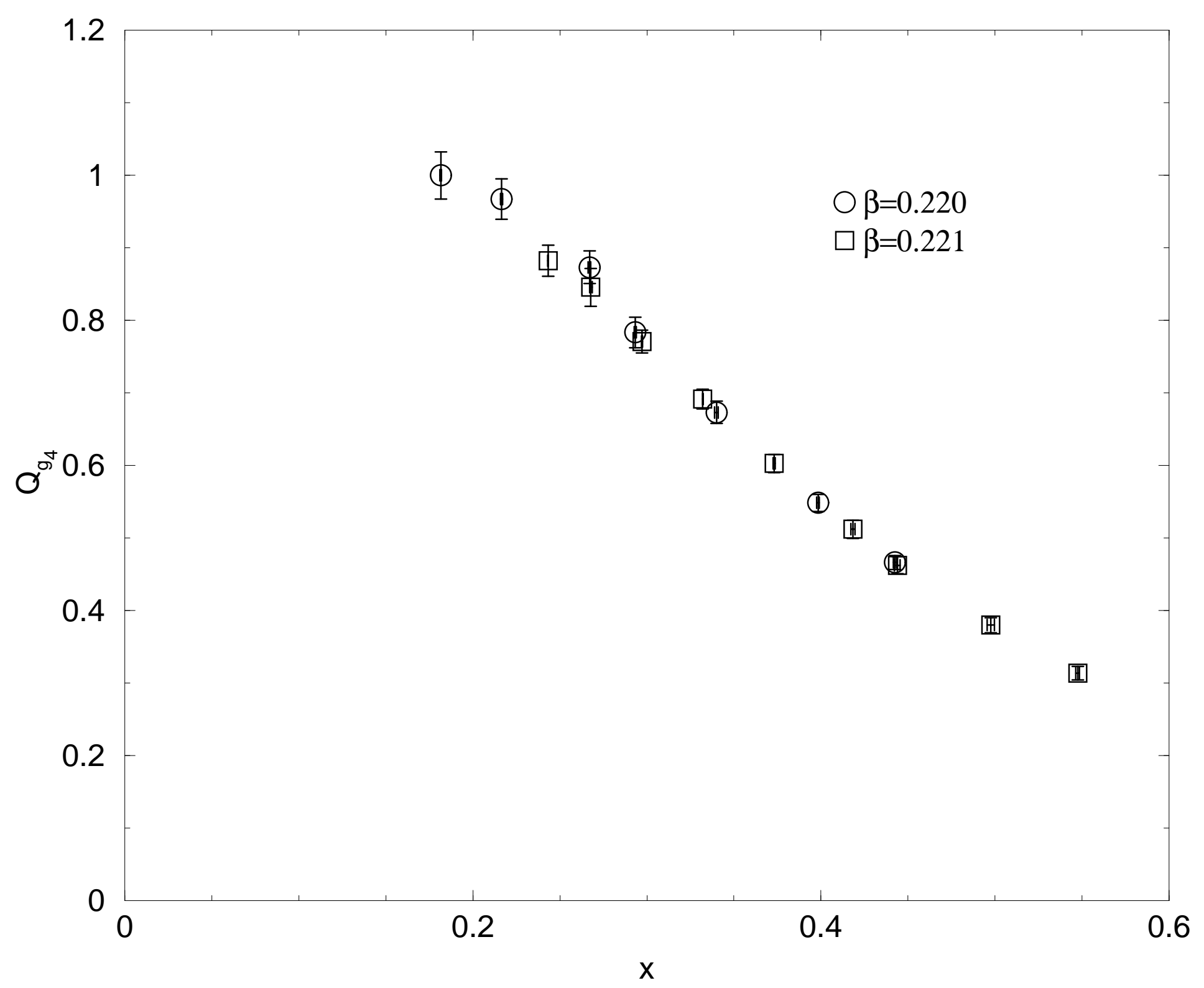




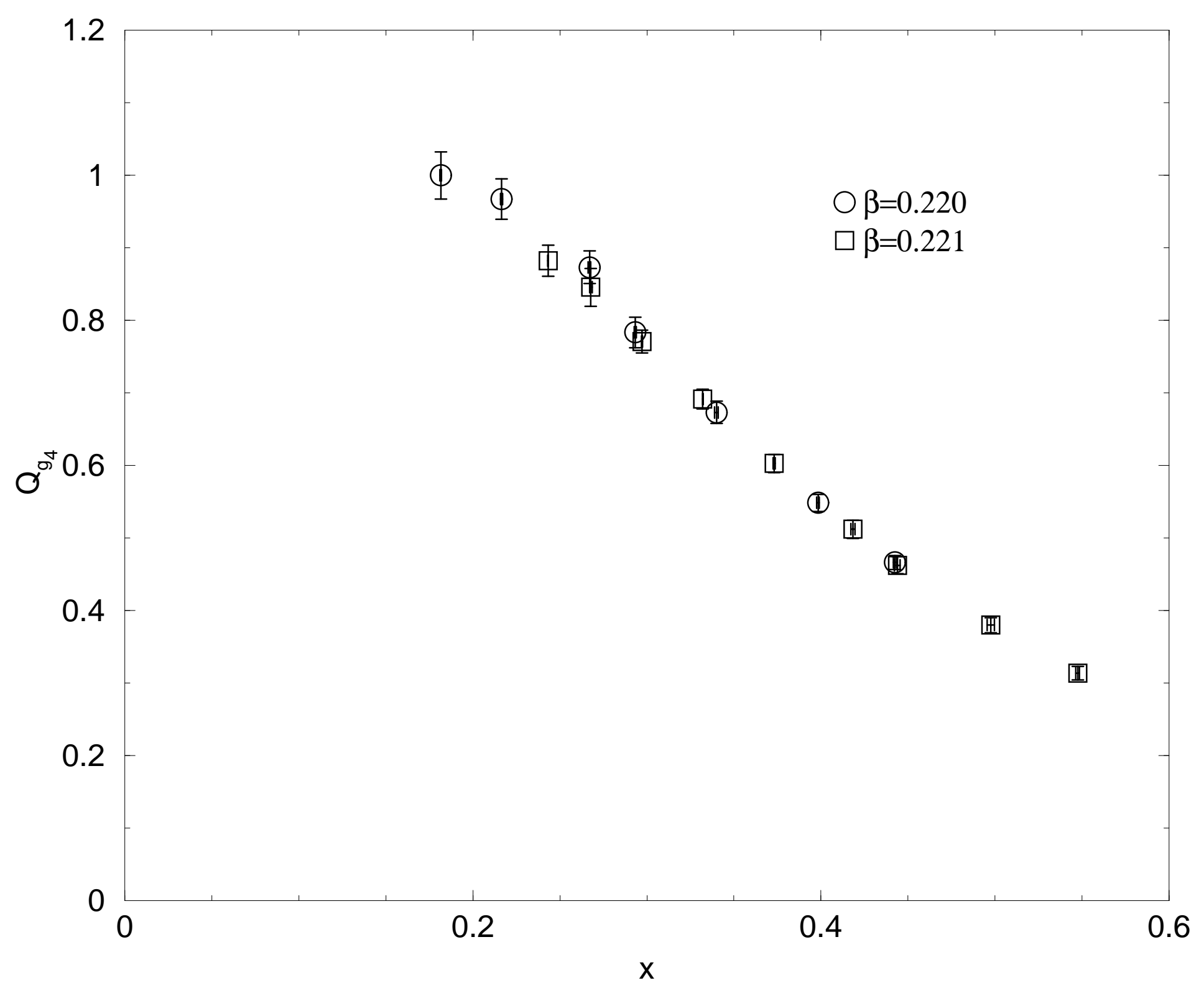

